\title{
Dog Bites: Hidden Danger of Fulminant Sepsis
}

\author{
Richard W. Tisovec and Ram Kakaiya, MD
}

Dog bites are a common problem in family practice. Although the summer months often show a seasonal increase in the number of bites seen, they can and do occur at any time during the year. Often the family pet, not a stray animal, inflicts the bite. Because many dog owners have their pets vaccinated and know whether their pet has been exposed to rabies, victims bitten by the family dog are likely to assume that they cannot be infected. This assumption, however, is not valid. The danger of not seeking medical attention for animal bites is that infection can occur even when caused by the most well-cared-for pet. The following is a case report of a patient who did not report a dog bite to his family physician, even at the initial signs and symptoms of systemic illness, because he thought the bite was unimportant and not related to his illness.

\section{Case Report}

A 59-year-old man with a history of hypertension, cigar smoking, and stabilized cardiac arrhythmia came to our health clinic complaining of shaking chills, myalgia, lethargy, left lower quadrant abdominal pain, nausea, vomiting, dry mouth, and diarrhea with mucus. He was taking digoxin, amiodarone, lovastatin, and a daily aspirin for his heart disease and his high-cholesterol levels. His surgical history included repair of right and left inguinal hernias, appendectomy, and coronary artery bypass graft following myocardial infarction.

His temperature was elevated to $102^{\circ} \mathrm{F}$, his blood pressure was $120 / 72 \mathrm{mmHg}$, and his heart rate was 104 beats per minute. He had diffuse tenderness, increased bowel sounds, and guarding with deep palpation, as well as point tenderness in the left lower quadrant with some rebound ten-

Submitted, revised, 14 October 1997.

From the University of Illinois College of Medicine at Rockford (RWT) and the Rockton Community Health Clinic, University of Illinois, Rockford (RK). Address reprint requests to Richard W. Tisovec, 4041 Renn Hart Hills Rd, Apt 20, Loves Park, IL 61111. derness, during an abdominal examination. His stool was positive for occult blood. An erythematous macular rash was noted over the patient's feet and legs. Laboratory studies showed a white cell count of $23,900 / \mu \mathrm{L}$ with 39 percent band forms, hemoglobin $16 \mathrm{~g} / \mathrm{dL}$, and a platelet count of $25,000 / \mu \mathrm{L}$.

He was admitted to the hospital later that day with a presumed diagnosis of diverticulitis and possible secondary sepsis. On admission his white cell count was $16,600 / \mu \mathrm{L}$ and his platelet count was $8000 / \mu \mathrm{L}$. The patient began a therapeutic regimen of ciprofloxacin and metronidazole before blood culture results could be obtained. A plain film and computed tomographic scan of the abdomen were unremarkable. The blood cultures grew a fine, tapered, gram-negative rod with growth characteristics suggesting an anaerobe. Intravenous antibiotics were then changed to clindamycin and ceftazidime.

A culture of the bacteria was sent to the Mayo Clinic in Rochester, Minn, for further study. The organism was identified as Capnocytopbaga cani-. morsus, a rare pathogen of $\operatorname{dog}$ bites. The patient, subsequently questioned by his family physician about contact with dogs, admitted to having been bitten 8 days before the onset of symptoms. When examined, the bite was unremarkable and appeared to have healed well. Ceftazidime therapy was stopped, the patient continued with clindamycin, and his symptoms resolved 1 week later. Fortunately, no long-term effects resulted from this patient's case of bacterial sepsis.

\section{Discussion}

$C$ canimorsus is a facultatively anaerobic gramnegative rod that prefers an environment of 5 percent carbon dioxide and is part of the normal oral flora of 16 percent of dogs. ${ }^{1,2}$ Human infections are rare but potentially fatal, and transmission of this bacteria is strongly associated with dog bites and the licking of open wounds or sores by dogs. ${ }^{1}$ Many of the dog bites sustained are not visibly in- 
fected and are seemingly insignificant. ${ }^{2}$ As of July 1996 only about 125 cases of infection with $C$ canimorsus have been reported worldwide. ${ }^{3}$ Symptoms are usually seen 7 to 10 days after infection, and the fatality rate associated with this disease is approximately 25 percent. ${ }^{1,3}$ Most who die are immunocompromised, although there have been fatalities associated with immunocompetent persons. ${ }^{3}$ Predisposing conditions associated with serious infection with this organism include splenectomy, alcoholism, trauma, Hodgkin disease, idiopathic thrombocytopenic purpura, corticosteroid therapy, chronic lung disease, peptic ulcer, hypertension, and atherosclerotic heart disease. ${ }^{1,3,4}$ In practice, antibiotic sensitivity is difficult to obtain for this organism because the bacterium grows slowly, and results are not available in a timely manner.

In this instance of dog bite infection, the transmitted organism was a rare species of bacteria transmitted from the oral flora of a healthy dog. The most common organisms transmitted by dog bite are Stapbylococcus aureus, Pasteurella multocida, and various streptococci. If a dog bite is reported, an antibiotic to which these organisms are sensitive should be prescribed. Common choices include amoxicillin-clavulanate or penicillin plus dicloxacillin. $P$ multocida is often resistant to such drugs as cephalexin and erythromycin. Initial therapy with a parenteral dose of the antibiotic can produce a rapid onset of therapeutic levels. Prescribing antibiotics prophylactically for bites that do not appear to be septic has been questioned in the past because of doubts about effectiveness and the time it takes for oral antibiotic therapy to achieve therapeutic levels (adjusting for the patient's delay in having a prescription filled and starting therapy). A meta-analysis published in 1994 found that risk reduction approximated 1 patient avoiding sepsis for every 14 taking antibiotics prophylactically. ${ }^{5}$

In conclusion, even the most astute family physician can overlook an animal bite simply by not inquiring about it or not observing it on physical examination, especially if the bite is small and has healed by the time the symptoms of sepsis occur. In those cases where a bite has been reported and rabies has been ruled out, other organisms inoculated by the bite can cause local infection and even sepsis. Such preventive measures as prophylactic antibiotic use, therefore, need to be taken to prevent possible hospitalization of these patients. Our case serves as a reminder that, because of the many organisms in the oral flora of animals, bites can become infected and progress to septicemia.

\section{References}

1. Griego RD, Rosen T, Orengo IF, Wolf JE. Dog, cat, and human bites: a review. J Am Acad Dermatol 1995;33:1019-29.

2. Forlenza SW. Capnocytophaga: an update. Clin Microbiol Newslett 1991;13:89-91.

3. Pers C, Gahrn-Hansen B, Frederiksen W. Capnorytophaga canimorsus septicemia in Denmark, 19821995: review of 39 cases. Clin Infect Dis 1996;23:71-5.

4. Linton DM, Potgieter PD, Roditi D, Phillips A, Adams BK, Hayhurst M, et al. Fatal Capnocytophaga canimorsus (DF-2) septicaemia. A case report. S Afr J Med 1995;84:857-60.

5. Cummings $P$. Antibiotics to prevent infection in patients with dog bite wounds: a meta-analysis of randomized trials. Ann Emerg Med 1994;23:535-40. 